\title{
Uso de las redes sociales como elemento formativo en el aula: Análisis de la motivación del alumnado universitario
}

\section{Use of social networks as a strategy formative in the classroom: Analysis of university students motivation.}

\author{
David Hortigüela Alcalá \\ Prof. Departamento de didácticas específicas. Área de expresión corporal \\ (Universidad de Burgos) \\ Ángel Pérez Pueyo \\ Prof. Área de educación física y deportiva \\ (Universidad de León)
}

Fecha de recepción: 23 de mayo de 2015

Fecha de revisión: 4 de junio de 2015

Para citar este artículo: Hortigüela Alcalá, D. y Pérez Pueyo, A. (2015):

Uso de las redes sociales como elemento formativo en el aula: Análisis de la motivación de alumnado universitario, Icono 14, volumen (13), pp. 95-115. doi: $10.7195 /$ ri14.v13i2.788 


\section{Resumen}

El presente estudio, realizado en la Facultad de Educación de Burgos en el curso escolar 2013-2014, analiza la percepción del alumnado universitario sobre la motivación hacia el aprendizaje que genera el uso de las redes sociales como estrategia metodológica. Han participado 187 alumnos de cuatro asignaturas, dos del Grado de Educación en Primaria (grupo A: uso NNTT) y dos del Grado en Terapia Ocupacional (grupo B: no uso NNTT), así como dos docentes. En las asignaturas del grupo $A$ se han utilizado las redes sociales como vía fundamental para el desarrollo de las actividades del alumno, mientras que en el grupo B el uso de estas herramientas no se ha llevado a cabo. La metodología de la investigación ha sido mixta, empleándose tanto un análisis cuantitativo (descriptivo e inferencial) como cualitativo (entrevistas). Se emplea un pretestpostest, comprobando en qué medida varía la motivación del alumnado tras haber cursado las asignaturas. Se observa cómo los estudiantes que ha utilizado las redes sociales como elemento formativo presentan una implicación hacia los contenidos significativamente mayor que aquellos que no lo han hecho. La edad y el uso de las redes sociales fuera del aula son las variables que presentan diferencias entre grupos sobre el bagaje de los contenidos adquiridos. Por otra parte, los docentes muestran una gran disparidad de opiniones respecto al uso formativo que pueden tener las redes sociales.

\section{Palabras clave}

Percepción docente - Redes sociales - Implicación del alumno - Motivación - Aprendizaje obtenido - Retroalimentación

\section{Abstract}

This study analyzes the perception of university students on motivation toward learning generated by the use of social networks as a methodological strategy. 187 students in four subjects have participated. In subjects in group A social networks were used as critical to the development of student activities, whereas in group $B$ the use of these tools has not been carried out. The research methodology has been mixed, using both a quantitative analysis (descriptive and inferential) and qualitative (interviews). A pretest-posttest is used, checking how varies the student motivation after the subjects. It shows how students who used social networks had an impli-

ICONO14 | Año 2015 Volumen 13 N² | ISSN: 1697-8293 | DOI: ri14.v13i2.788 
cation toward contents significantly higher than those who have not done. The age and the use of social networks out of classroom are the variables that differ between groups on the acquired content. Moreover, teachers show a wide disparity of views on the educational use of social networks.

\section{Key Words}

Teacher perception - Social network - Student engagement - Motivation - Learning obtained - Feedback

\section{Introducción}

En la última década ha aumentado exponencialmente el uso de las redes sociales en nuestro país, superando con creces la media de la población europea (Monsalve \& Granada, 2013). Tanto es así, que el $66 \%$ de los españoles utiliza asiduamente este medio de comunicación, mientras que en Europa ronda el 40\%. Estos datos se asocian a otros, impensables hace 20 años, como que hoy en día existen más teléfonos móviles que habitantes en España, con una tasa de penetración del 118\% según el informe Social, Digital \& Mobile in Europe en el año 2014.

Esta situación refleja claramente un cambio de paradigma en el ámbito relacional del individuo, derivando en una forma diferente de comunicarse, buscar información y proceder en cuanto a las actuaciones y comportamientos de los alumnos en las actividades cotidianas (Low, Polanin \& Espelage, 2013). El sistema educativo, y sobre todo la manera de concebir la educación, no debe permanecer inmune ante este profundo cambio, tratando de adaptarse a los nuevos intereses del alumno influidos por esta nueva concepción social (Amirault, 2012). Será por lo tanto fundamental diseñar estrategias metodológicas que favorezcan y permitan la utilización de las tecnologías de la información y de la comunicación (TIC) en el aula desde un enfoque formativo y basado en el aprendizaje (Martínez- González, 2013). A partir de esta última idea surge una cuestión trascendente y nada fácil de responder: ¿Cómo hacer que el alumno utilice las redes sociales con criterio racional y un carácter educativo? Que el alumnado dedica tiempo a las redes sociales es evidente, pero lo relevante es saber cuánto de este tiempo es excesivo y cómo podemos invertir el mismo hacia actividades formativas y que fomenten aprendizaje (Cappella, Neal \&

DOI: ri14.v13i2.788 | ISSN: 1697-8293 | Año 2015 Volumen 13 N² | ICONO14 
Sahu, 2012). Por lo tanto, parece lógico entender los beneficios que puede conllevar el uso de las redes sociales, ya que negarlo supondría limitar un uso de herramientas que los alumnos utilizan asiduamente fuera del aula (Araujo, Fruchter, Garbín, Nóbilo \& Arantes, 2014). Del mismo modo, autores cómo Ormart y Navés (2014) reflejan experiencias en las que se relaciona directamente el uso de las redes sociales con fines formativos dentro del aula con una mayor independencia en la búsqueda de información del alumno y una mayor autonomía en la regulación de su aprendizaje. En esta línea, y entendiendo la riqueza metodológica que otorga el hecho de compartir experiencias, ideas y proyectos con otros miembros de la comunidad educativa, la utilización de las redes sociales favorece la creación de vínculos formativos que incidan en el tratamiento de una temática determinada. Como establece Toetenel (2014) la principal ventaja es que el alumno tiene la posibilidad de colgar información en el momento que quiera, mediante cualquier tipo de dispositivo $\mathrm{y}$ en el formato que prefiera. Estos tres factores proyectan un amplio abanico de posibilidades que se escapan al contenido tradicional impartido en el aula y en un horario determinado. Además, actualmente los estudios semipresenciales u online han aumentado exponencialmente en los últimos años, lo que hace que irrevocablemente los docentes deban pensar en el uso de otro tipo de metodologías que den alternativa a los enfoques más tradicionales (Windes \& Lesht, 2014).

Si en la etapa educativa obligatoria en muchas ocasiones el rol del docente y el de la comunidad educativa ha de centrarse en el control y la limitación del uso de las redes sociales, en el ámbito universitario los esfuerzos habrán de dirigirse en la búsqueda de estrategias metodológicas y evaluativas que favorezcan su utilización (Al-Mukhaini, Al-Qayoudhi, \& Al-Badi, 2014). No se trata de abordar la enseñanza de su uso, sino de enfocar su trabajo como medio en la difusión, tratamiento y profundización de los contenidos que se abordan en clase (García \& Martín, 2013). Abe y Jordan (2013) reflejan la riqueza que tiene el uso de las redes sociales (abordadas desde un punto de vista racional y crítico) como elemento reflexivo dentro del aula, ya que permiten obtener diferentes puntos de vista sobre aspectos que si solamente se leyeran en unos apuntes sería inviable. Por ello, Lu y Churchill (2014) recomiendan que este uso tecnológico de carácter formativo se asocie a aspectos de enseñanza como la búsqueda óptima de información, la conciencia crítica sobre lo que se lee y la extracción de conclusiones significativas cada vez que se utilice.

ICONO14 | Año 2015 Volumen 13 N² 2 | ISSN: 1697-8293 | DOI: ri14.v13i2.788 
Son varias las experiencias (Barrientos \& Mogollón, 2013; Martínez-Berruezo \& García-Varela, 2013; Pérez-Fernández \& Vílchez, 2013) que se fundamentan en la utilización de las redes sociales en el aula, demostrando que lo importante no es el nivel que los alumnos adquieren en dicho uso, sino el modo en el que el docente orienta el proceso para obtener un mayor aprendizaje. Esta regulación en su tratamiento podrá garantizar la obtención de beneficios en términos educativos. Román, Cardemil y Carrasco, (2012) reflejan que las TIC no son buenas o malas de por sí, sino que el tratamiento que se haga de las mismas y el autocontrol del individuo que las utiliza definirán sus pros y contras en ese caso concreto. De lo que no hay duda es de la profundización en la información que conlleva su utilización, lo que las convierte en un eje de acción esencial que ha de ser guiado por el docente independientemente de la asignatura que se trate.

Desde el punto de vista metodológico, y bajo el prisma de actuación de la utilización de enfoques didácticos en las asignaturas que permitan al alumno implicarse en su aprendizaje, son de sobra conocidos los PLE (personal learning environment), donde el discente puede construir su propio entorno de aprendizaje bajo criterios de control, autogestión y de manera compartida. Este enfoque por proyectos (cada vez más habitual en el ámbito universitario) favorece un visionado más amplio de los propios contenidos de asignaturas concretas, centrándose en la adquisición de competencias transversales (Marín, Salinas \& De Benito, 2014). Además implica mecanismos de actuación cooperativos entre los integrantes del aula, alejándose así de enfoques más tradicionales e individualistas. Otro de los aspectos que pueden favorecer las TIC, y en concreto las redes sociales, es el feedback entre profesor y alumno, haciendo visible todo aquello que los participantes quieran compartir, debatir...Esto se asocia al reflejo de evidencias de aprendizaje cuando el alumno se encuentra conectado, lo que puede derivar en la proposición de sistemas de evaluación por parte del docente que garanticen la participación del alumno (García-González, 2014).

Por lo tanto, es positivo, siempre que se delimiten unos criterios definidos de actuación, establecer mecanismos evaluativos a lo largo del proceso que garanticen el guiado y la orientación del aprendizaje del alumno. Balakrishnan (2014) indica que si los estudiantes utilizan estas herramientas fuera del aula el entorno

DOI: ri14.v13i2.788 | ISSN: 1697-8293 | Año 2015 Volumen 13 N² 2 | ICONO14 
educativo ha de aprovechar este hecho con fines de aprendizaje. En el caso de las redes sociales, divulgar información, compartir experiencias, y sobre todo analizar enfoques de la misma temática que tienen otros profesionales, pude enriquecer sin duda el proceso de enseñanza y aprendizaje.

En este sentido, en este estudio se comprueba el nivel de influencia que tiene el uso de las redes sociales en la motivación del alumno y en su implicación hacia los contenidos abordados, comparando esto con grupos que no han utilizado las TIC dentro la asignatura. Esta comparación entre grupos bajo el enfoque de dos metodologías diferentes es algo sobre la que la bibliografía no ha incidido demasiado.

\section{Material y métodos}

\subsection{Objetivos}

- Comparar la percepción que tiene el alumnado sobre la motivación hacia el aprendizaje y la implicación en los contenidos entre los grupos que han utilizado las redes sociales como elemento formativo y los que no.

- Analizar el grado de influencia que tiene las variables de edad, curso y nivel de uso de las redes sociales fuera del aula en la percepción del alumno sobre el bagaje de conocimientos adquiridos.

- Contrastar las opiniones que tienen los docentes sobre los beneficios y/o desventajas que conllevan la utilización o no de las redes sociales como herramienta de fomento del aprendizaje.

\subsection{Participantes}

Para este estudio se ha empleado una muestra de 187 participantes (61,3\% mujeres y $30.7 \%$ hombres), con una media de edad de 21.47 años (DT $=2.31$ ). Toda la muestra que ha cumplimentado el cuestionario forma parte de 4 asignaturas impartidas en el cuso escolar 2013-2014 en la Facultad de Educación de Burgos. Pertenecen a 2 grados: Primaria (grupo A, 91 alumnos) y Terapia Ocupacional (gru-

ICONO14 | Año 2015 Volumen 13 N² 2 | ISSN: 1697-8293 | DOI: ri14.v13i2.788 
po B, 96 alumnos). El grupo A ha utilizado en las dos asignaturas de Primaria las redes sociales como elemento formativo, mientras que el B no. Las dos asignaturas del grupo A son impartidas por un docente (29 años y 4 años de experiencia) y las dos del grupo B por otro (58 años y 28 años de experiencia). Tras realizar la prueba de SHAPIRO-WILK se observa que la muestra responde a parámetros de normalidad $(\mathrm{p}=.113)$. En la tabla 1 pueden observarse los datos relativos al número de los alumnos que integraba cada uno de los grupos.

\begin{tabular}{|c|c|c|c|c|}
\hline ASIGNAURA & GRADO & CURSO & USO TIC & $N^{\circ}$ DE ALUMNOS \\
\hline $\begin{array}{c}\text { Educación Física y su } \\
\text { Didáctica }\end{array}$ & Primaria & $2^{\circ}$ & Sí (GRUPO A) & 55 \\
\hline Juego Educativo & Primaria & $4^{\circ}$ & Sí (GRUPO A) & 36 \\
\hline $\begin{array}{c}\text { Actividad Física y } \\
\text { salud }\end{array}$ & T. Ocupacional & $2^{\circ}$ & NO (GRUPO B) & 59 \\
\hline Psicomotricidad & T. Ocupacional & $4^{\circ}$ & NO (GRUPO B) & 37 \\
\hline
\end{tabular}

Tabla 1: Participantes en el estudio (asignatura, grado, uso TIC y número de participantes en cada asignatura.

\subsection{Metodología}

En este apartado incidiremos en el tipo de metodología que se ha empleado en el estudio, destacando las técnicas propias del análisis realizado. También se especificarán los participantes de la investigación así como los instrumentos de recogida de datos llevados a cabo. Por último, se abordará el punto de diseño y procedimiento en el que se detallarán las características de los dos grupos generados en función del uso de las redes sociales.

\section{Análisis empleado}

La metodología de la investigación ha sido mixta, empleándose tanto un análisis cuantitativo (descriptivo e inferencial) como cualitativo (entrevistas). Esta complementariedad en el tratamiento de los datos otorgará tanto un visionado más global de los resultados obtenidos como una mayor comprensión de los mismos (Schram, 2014). Se emplea un pretest-postest, comprobando en qué medida varía la motivación del alumnado tras haber cursado las asignaturas. 


\section{Cuantitativo}

Se lleva a cabo un tratamiento descriptivo, (medias y DT) y uno inferencial (Anovas) para cada uno de los dos grupos. El tratamiento de los datos en el pretestpostes se hace a través de los factores de análisis, viendo si existen diferencias significativas en los factores entre grupos antes y después de haberse llevado a cabo la asignatura. En el ANOVA se observa si existen diferencias intragrupales para las tres variables independientes utilizadas.

\section{Cualitativo}

El análisis cualitativo se realizó a partir de la recogida estructurada de información mediante una entrevista a los docentes participantes en la investigación. Los datos extraídos se analizaron a través del análisis de la temática del contenido (Libarkin y Kurdziel, 2002) y la comparación constante entre los datos (Denzin y Lincoln, 1994). El análisis del contenido se centró en la búsqueda de patrones en el texto, codificando los extractos coincidentes con los patrones cruzados (Saldaña, 2009). Los temas que surgieron en el primer análisis independiente fueron examinados críticamente por todos los investigadores a través de un diálogo reflexivo. La confiabilidad fue apoyada a través de la retroalimentación continua y el análisis participativo por parte de los investigadores que revisaron y perfeccionaron las categorías emergentes, para que los resultados pudieran ser considerados confiables, creíbles y transferibles (Lincoln y Guba, 1985). El objetivo era utilizar la información obtenida para dotarla de una mayor comprensibilidad a través de la transferencia de los resultados. Las categorías que surgieron de los datos se presentan de forma explícita a través de la sección de resultados y con el apoyo de varios ejemplos de textos (Cohn, 1991).

Para recapitular, organizar y obtener la saturación de los datos a partir de las categorías generadas en las preguntas realizadas a los dos docentes se ha utilizado el programa de computación WEFT QDA. El acrónimo empleado para el docente que utilizó las redes sociales es DRS (docente redes sociales) y para el que no las utilizó es DNR (docente no redes).

ICONO14 | Año 2015 Volumen 13 Nº 2 | ISSN: 1697-8293 | DOI: ri14.v13i2.788 


\subsection{Instrumentos de recogida de información utilizados}

\subsubsection{Cuantitativos}

Como instrumento de recogida de datos se ha utilizado el cuestionario validado de evaluación en formación inicial (Castejón, Santos \& Palacios, 2013). Para ello, y con el fin de obtener la garantía en la percepción del alumnado sobre el aprendizaje recibido y la implicación hacia los contenidos desarrollados, el estudiante cumplimentó específicamente la Escala de Sistemas de Evaluación. Esta escala tiene un total de 18 cuestiones a las que los discentes responden en grado al acuerdo con el enunciado en una escala tipo Likert, cuyos valores van desde 1 (Nada) hasta 5 (Mucho). El cuestionario presenta una fiabilidad del Alpha de Cronbach de 0.831, superior al límite inferior que según Corbetta (2007) es aceptado como fiable. Se aplica un nivel de confianza del $95 \%$. Además, esta escala se construye a partir de la literatura especializada en el ámbito universitario, siendo validada por un grupo de expertos y existiendo una relación entre los ítems de la misma y los objetivos de la presente investigación.

Para el cuestionario final se realizó un Análisis Factorial de Componentes Principales, donde se obtienen valores adecuados para el índice KMO de 0.807 como en el test de esfericidad de Barlett $(p>.00)$. Los índices obtenidos en la matriz de covarianzas, presentaron ajustes satisfactorios para el índice RMSEA $=0.078$, como en el GFI $=0.88$. Así, se obtienen 2 factores que integran el cuestionario:

1. Motivación hacia el aprendizaje (8 ítems): se abordan ítems relacionados con la estimulación que presenta el alumno hacia las tareas recibidas, así como la autonomía y autorregulación a lo largo de la asignatura.

2. Implicación hacia los contenidos (10 ítems): se atiende al grado de continuidad y seguimiento que el estudiante muestra en el proceso, reflejando una predisposición positiva hacia las tareas que se proponen. 


\subsubsection{Cualitativos}

La extracción de información cualitativa de la investigación se realizó a través de una entrevista semi-estructurada a los dos docentes participantes en la misma. El objetivo era explorar los pensamientos y las sensaciones de los entrevistados para obtener una perspectiva interna de la experiencia (Patton, 2002). En base a esta idea, se desarrolló un guión tomando como referencia los conocimientos previos de los investigadores y trabajos similares de investigación. Como la naturaleza de la entrevista era semi-estructurada, los investigadores pudieron añadir nuevas preguntas en base a las respuestas de los entrevistados para convertir la entrevista en una conversación (Patton, 2002). Este formato abierto permite explorar nuevas áreas para producir datos más ricos (Smith \& 0sborn, 2003). Atendiendo a las variables del estudio se preguntaron cuatro cuestiones principales a cada uno de los docentes al finalizar las asignaturas;

1. ¿Qué factores consideras relevantes para que el alumno se motive hacia lo que aprende?

2. ¿Qué estrategias metodológicas utilizas para conseguir una mayor implicación hacia los contenidos?

3. ¿Cómo enfocas la evaluación a lo largo del proceso?

4. ¿Consideras que el uso de las TIC y las redes sociales pueden favorecer la motivación del alumno por la asignatura?

Toda la información relativa a estas respuestas se ha agrupado en tres categorías:

1. “Motivación e implicación del alumnado hacia el aprendizaje"

2. "Estrategias metodológicas utilizadas"

3. "Enfoque evaluativo a lo largo del proceso" 


\subsection{Diseño y procedimiento del estudio}

Las cuatro asignaturas que componen la muestra tienen un carácter semestral. El estudio es de carácter retrospectivo, ya que el alumnado ha cumplimentado el cuestionario una vez ha finalizado las asignaturas y conoce su calificación. A continuación se expone el enfoque metodológico que los docentes han dado a las asignaturas en cada uno de los grupos:

- Grupo A uso de las TIC: se utilizan las redes sociales (twitter e Instagram) como elemento formativo. Los alumnos comparten información mediante el establecimiento de un hashtag en la asignatura. Del mismo modo suben imágenes que sirven de evidencias de la realización de las tareas. Este proceso es seguido por el docente, que lleva a cabo un seguimiento en el aula virtual de todo lo desarrollado, otorgando un feedback a lo largo del proceso. El alumno profundiza en la información que se ha trabajado en clase, y por grupos se suben diferentes vídeos, links de páginas web u opiniones de expertos en la temática abordada que permitan generar debates constructivos.

- Grupo B no uso de las TIC: No se utilizan las TIC ni las redes sociales. El enfoque es más tradicional y es el docente el que además de proponer las tareas a realizar, facilita el material y los recursos que el estudiante ha de utilizar. El análisis del contenido se realiza en las clases y no suele asociarse con una búsqueda de profundización por parte del alumno mediante el uso de internet. El alumno trabaja en grupos, pero siempre de manera presencial, lo que provoca que no siempre existan las oportunidades necesarias para generar debates entre los miembros de la clase sobre lo trabajado.

Al finalizar la asignatura, los estudiantes respondieron de forma individual en el aula al cuestionario en una única sesión de una hora de duración. En todo momento se garantizó el anonimato para que las respuestas de los estudiantes fueran lo más sinceras posibles, al igual que se garantizó la confidencialidad en el tratamiento de los datos.

DOI: ri14.v13i2.788 | ISSN: 1697-8293 | Año 2015 Volumen 13 N² 2 | ICONO14 


\section{Resultados}

\section{Análisis cuantitativo: descriptivo}

\begin{tabular}{|c|c|c|c|c|c|c|c|}
\hline & \multicolumn{4}{|c|}{ PRE-TEST } & \multicolumn{3}{|c|}{ POST-TEST } \\
\hline & $\mathrm{N}$ & Media & DT & Var. & Media & DT & Var. \\
\hline \multicolumn{8}{|c|}{ Grupo uso TIC (a) } \\
\hline F.1. Motivación & 91 & 3,95 & .201 & .040 & 4,22 & .186 & .034 \\
\hline F.2. Implicación & 91 & 3,62 & .197 & .038 & $4,51^{\text {aa }}$ & .231 & .053 \\
\hline \multicolumn{8}{|c|}{ Grupo no uso TIC (b) } \\
\hline F.1. Motivación & 96 & 4,01 & .217 & .047 & 3,94 & .295 & .087 \\
\hline F.2. Implicación & 96 & 3,96 & .341 & .011 & $3,53^{\text {ba }}$ & .176 & .030 \\
\hline
\end{tabular}

Nota: Los superíndices reflejan los grupos entre los que se encuentran las diferencias significativas a nivel .05

Tabla 2: Comparación de medias por factores para cada uno de los grupos en el pretest-postest (nivel de significación en las diferencias: ${ }^{*} p<.05$ )

Se observa cómo en el pretest no existen diferencias significativas en ninguno de los factores entre grupos, alcanzándose incluso medias más elevadas en el grupo que no utilizó las TIC (los profesores es la primera vez que dan clase a estos alumnos). Al finaliza la asignatura, las medias de los dos factores se incrementan en el grupo A, mientras que en el B se reducen. En el factor de implicación hacia los contenidos existen diferencias significativas en el grupo A entre el pretest-postest y entre los dos grupos al finalizar la asignatura.

\section{Análisis inferencial: ANOVAS}

BONFERRONI Y POST HOC. A partir del análisis factorial realizado y en relación a los ítems relacionados con el aprendizaje desarrollado, se ha creado la variable de escala denominada "bagaje de conocimientos adquiridos en la asignatura". Para ello se realiza un Anova de una vía para grupos independientes, con el fin de comprobar si existen diferencias estadísticamente significativas acerca del aprendizaje generado, atendiendo a las variables independientes de edad, curso y el uso de redes sociales fuera del aula. Además se realiza un post hoc indicando en qué grupos se encuentra esa diferencia significativa. La variable de edad se categoriza en: 1- "entre 20 y 22", 2- "entre 23 y 25" y 3- "más de 25". La de curso en: 1- "se- 
gundo", 2- "tercero" y 3 "cuarto". La de uso de redes sociales fuera del aula en: 1- "asiduamente", 2- "de manera esporádica" y 3- "no uso". (tabla 3).

\begin{tabular}{|c|c|c|c|}
\hline $\begin{array}{c}\text { BAGAJE DE } \\
\text { CONOCIMIENTOS } \\
\text { ADQUIRIDOS }\end{array}$ & $F$ & $g l$ & $p$ \\
\hline \multicolumn{4}{|c|}{ Grupo uso TIC (a) } \\
\hline Edad & 109.34 & 1 & $.023^{*}$ \\
\hline Curso & 92.42 & 2 & .213 \\
\hline Uso de redes sociales & 67.66 & 1 & .142 \\
\hline \multicolumn{4}{|c|}{ Grupo no uso TIC (b) } \\
\hline Edad & 114.26 & 1 & .297 \\
\hline Curso & 96.78 & 2 & .145 \\
\hline Uso de redes sociales & 69.71 & 1 & $.013^{* *}$ \\
\hline
\end{tabular}

${ }^{*} p<.05$ entre 20-22 años (media 4.67) y más de 25 años (media 3.81)

${ }^{*} p<.05$ entre asiduamente (media 4.21) y nunca (media 3.33)

Tabla 3: Resumen de Anova (Bonferroni) para cada una de las variables independientes analizadas en el postest (centro educativo, $n^{\circ}$ de alumnos clase y curso)

En el grupo que ha utilizado las redes sociales se obtienen diferencias significativas en la variable de la edad, siendo los alumnos más jóvenes los que consideran que la metodología abordada permite un mayor bagaje de los conocimientos adquiridos ( $F$ $(91)=109.34, p=.023)$. En el grupo que no ha utilizado las redes sociales las diferencias se encuentran en la variable del uso de las mismas fuera del aula, y los que las utilizan asiduamente son los que más valoran el aprendizaje obtenido ( $F(96)=69.71, \mathrm{p}=.013$ ).

\section{Análisis cualitativo}

De toda la información extraída de las respuestas de las entrevistas realizadas a los docentes participantes surgieron tres temas o categorías fundamentales:

1. Motivación e implicación del alumnado hacia el aprendizaje

2. Estrategias metodológicas utilizadas

3. Enfoque evaluativo a lo largo del proceso

DOI: ri14.v13i2.788 | ISSN: 1697-8293 | Año 2015 Volumen 13 N² 2 | ICONO14 
Motivación e implicación del alumnado hacia el aprendizaje: Se observa cómo los dos docentes tienen una percepción dispar sobre la manera en la que el alumno puede motivarse hacia los contenidos impartidos, debido en parte a los fines educativos que priorizan en las asignaturas: "Es tan importante el contenido en sí como la manera de abordarlo. Un contenido puede ser denso de por sí, pero si se presenta al alumno desde un enfoque más interesante su motivación puede aumentar" (DRS). "No podemos pretender que el estudiar sea siempre motivante [...]" "Habrá alumnos a los que les guste más que otros, pero los contenidos hay que impartirlos, y eso es indiscutible" (DNR).

Estrategias metodológicas utilizadas: Se observa cómo no solamente las estrategias utilizadas son diferentes, ya que la relevancia que el profesor otorga a las mismas influye también en su modo de dar las clases: "La metodología es uno de los aspectos más fundamentales dentro del proceso educativo [...]" "Yo cada año intento modificar algún aspecto e incluir formas de actuación innovadoras en el aula, como este años las redes sociales" (DRS). "No creo que haya que volverse loco y cambiar la forma de impartir las clases. Yo llevo mucho tiempo utilizando la mismas metodología y no pienso cambiarla" [...] "Personalmente estoy contento y creo que mis alumnos también" (DNR).

Enfoque evaluativo a lo largo del proceso: Se aprecia como el docente del grupo $B$ centra la evaluación en la mera calificación, mientras que el del grupo A la entiende como un proceso formativo que garantiza el aprendizaje:

Es importante que el docente tenga claro el sistema de evaluación y de calificación que va a utilizar en su asignatura [...]" "Por ejemplo el uso de las redes sociales no lo califico, pero sí que evalúo el trabajo que realiza cada uno de los grupos, algo que me da una gran información. (DRS).

El alumno tiene que ganarse la nota, y para ello tiene que superar todas las pruebas [...]Yo hago pruebas muy variadas y la nota final es la media de todas ellas, creo que es lo más justo (DNR). 


\section{Discusión}

Se ha comprobado cómo el hecho de utilizar las redes sociales como elemento formativo produce en el alumno una mayor motivación, siendo la implicación hacia los contenidos impartidos el factor en el que se encuentran diferencias significativas entre grupos al finalizar las asignaturas. Los alumnos de menor edad, en el grupo que utilizaron las redes sociales, percibieron un mayor bagaje de los conocimientos adquiridos, algo que también sucedió en el grupo B para aquellos que sí que lo hacían fuera del aula. Entre los dos docentes existen discrepancias sustanciales en cuanto a la metodología utilizada, el sistema de evaluación empleada y las herramientas puestas en práctica para alcanzar la motivación en el alumno.

Que en el pretest no existan diferencias significativas entre grupos en ninguno de los factores indica unos índices de motivación similares hacia las asignaturas cursadas hasta el momento. Esta valoración se hace sobre cursos anteriores, sin atender a experiencias concretas cursadas en determinados cursos. Que la percepción inicial de los alumnos sea homogénea supone un aspecto positivo para la investigación, ya que atribuye que los resultados finales sean debidos a la intervención y/propuesta planteada en el estudio (Waldrip et al. 2014). Que las medias de los dos factores (motivación e implicación hacia los contenidos) se incrementen cuando finalizan las asignaturas en el grupo que utilizó las redes sociales, parece demostrar que su uso formativo dentro del aula puede tener beneficios en la implicación del alumnado hacia las tareas. Experiencias previas en esta línea (Aragón, AlDoubi, Kaminski \& Anderson, 2014) reflejan cómo la clave se encuentra en el uso racionalizado y guiado por parte del docente, siempre bajo la delimitación de premisas claras de intervención y focalizando los ejes temáticos que se pretenden abordar. Cabero-Almenara y Marín (2014) indican que la integración de las redes sociales en el aula ha de ser un complemento de aquello que se esté trabajando en clase, debiendo estar relacionado con una metodología que de continuidad al trabajo realizado y a aquello que se va a evaluar. Además la clave se encuentra en el tipo de información que se trate, de dónde provenga y el tipo de feedbcak existente tanto entre los miembros de la clase como de otros usuarios de la red (Madill, Gest \& Rodkin, 2014). En relación al feedback o retroalimentación, Jacquemin, Smelser y Bernot (2014) establecen que el docente ha de valorar y tener en cuenta

DOI: ri14.v13i2.788 | ISSN: 1697-8293 | Año 2015 Volumen 13 № 2 | ICONO14 
que en el caso de las redes sociales éste vendrá de varias fuentes: del profesor, de los propios compañeros y de otros miembros de la comunidad educativa presentes en la red, por lo que será preciso filtrar la información que se pueda generar. Además es necesario que el alumno adquiera códigos de respeto en las opiniones, así como en la formulación de intervenciones y asunción de críticas.

Es la implicación hacia los contenidos el factor en el que se obtienen diferencias significativas al finalizar la asignatura (tanto entre grupos como en el grupo que utilizó las redes sociales), lo que refleja una mayor continuidad y seguimiento del alumno a lo largo del proceso. Es preciso aclarar que este dato puede que no solamente sea achacado al uso de las redes, sino a la metodología asociada a la implementación de las mismas. En este sentido Fernández-Delgado (2012) muestra que es necesario controlar por parte del docente todo aquello que gira alrededor de la integración de su uso como elemento formativo, ya que pueden tener la consecuencia de una mayor implicación del alumno en su utilización, pero que sin embargo ésta no se encuentre asociada a los fines y objetivos delimitados en la asignatura. Por ello, Moral y Villalustre (2012) establecen algunas recomendaciones como la intervención del docente de manera asidua, el cambio de temática frecuente y el tratamiento de contenidos de manera presencial en el aula cuando se observe que el alumno se encuentra desorientado o hace aportaciones con poco criterio.

Dentro del grupo que utilizó las redes sociales se encontraron diferencias en función de la edad, siendo los alumnos más jóvenes los que percibieron un mayor bagaje de los conocimientos. Estudios como el de English y Carstensen (2014) establecen que el factor edad es clave dentro del uso de las redes sociales en el ámbito universitario, sobre todo cuando éste se enfoca a un uso sistemático que favorece la interrelación constante entre los usuarios. También existen experiencias (Barker, 2012) que demuestran un uso más racional y controlado en aquellos alumnos de mayor edad y acostumbrados a otras metodologías de trabajo. Dentro del grupo que no las utilizó en la asignatura las diferencias se encuentran en la variable del uso de las mismas fuera del aula, obteniendo de aquellos que si lo hacen una percepción más favorable sobre el aprendizaje obtenido. Este hecho se asocia a que los alumnos que las utilizan en el día a día lo hacen de manera voluntaria, lo que

ICONO14 | Año 2015 Volumen 13 N² 2 | ISSN: 1697-8293 | DOI: ri14.v13i2.788 
provoca una valoración positiva aunque no las hayan utilizado como herramienta formativa dentro del aula.

En relación a la parte cualitativa del estudio, se observa una discrepancia sustancial entre los dos docentes en cada una de las categorías generadas de análisis. El docente del grupo A considera que es tan importante el contenido como la manera de abordarlo, otorgando un rol esencial a la metodología desarrollada en el aula. Sin embargo el docente del grupo B presenta una opinión más utilitarista, valorando la importancia que tiene el contenido en sí y abogando por un único modelo a la hora de dar clase. Respecto al enfoque evaluativo utilizado a lo largo del proceso, se percibe cómo el docente del grupo A otorga a la asignatura una perspectiva fundamentada en la evaluación formativa, mientras que el del B, asocia directamente la evaluación con la calificación. En esta línea García-Valcárcel, Muñoz-Repiso, Hernández-Martín y Recamán (2012) determinan la relación directa que existe entre la metodología utilizada por el docente en el aula y la percepción que tenga el alumno sobre los aprendizajes obtenidos, siendo más favorables cuando tienen un carácter abierto y participativo. Por lo tanto en el presente estudio, tanto el uso de las redes sociales en el aula como la metodología empleada por el docente se determinan cómo influyentes en los resultados obtenidos.

\section{Conclusiones}

En relación al primer objetivo se ha demostrado como los alumnos que cursaron las asignaturas en las que se utilizaron las redes sociales obtuvieron una mayor motivación hacia el aprendizaje y más implicación hacia los contenidos abordados.

Respecto al segundo objetivo, dentro del grupo que utilizó las redes sociales se encontraron diferencias en función de la variable edad, siendo los alumnos más jóvenes los que valoraron en mayor medida el aprendizaje obtenido. Sin embargo, en el grupo que no las utilizó, las diferencias se encontraron en función de la variable de su uso fuera del aula.

En relación al tercer objetivo, ha quedado reflejado cómo los dos docentes manifiestan diferentes percepciones en las tres categorías generadas, relacionadas con-

DOI: ri14.v13i2.788 | ISSN: 1697-8293 | Año 2015 Volumen 13 N² 2 | ICONO14 
cretamente con las estrategias metodológicas empleadas y el sistema de evaluación puesto en práctica.

Consideramos el presente artículo de interés para todos aquellos docentes interesados en el uso de las TIC en el aula como elemento formativo, en concreto de las redes sociales. En este caso se llevó a cabo el uso de Twitter e Instagram, pero pueden utilizarse otras vinculadas al ámbito educativo como Edmodo, Iversity, Diipo o RedAlumnos. Por tanto, también puede ser de utilidad su lectura para aquellos profesores que consideran la metodología como piedra angular del proceso de enseñanza y aprendizaje, valorando la opción de integrar este tipo de propuestas en sus clases.

Este artículo presenta algunas limitaciones. En primer lugar únicamente valora a alumnos de la Facultad de Educación, por lo que sería necesario contrastar estos datos con asignaturas de otras especialidades que utilicen las redes sociales. En segundo lugar sería interesante analizar la percepción de alumnos de una misma asignatura en el que un grupo haya utilizado las redes sociales y otro no. De este modo los contenidos abordados serían los mismos y únicamente variaría el uso de esta herramienta formativa.

\section{Referencias}

Abe, P. , \& Jordan, N. (2013). Integrating Social Media into the Classroom Curriculum. About Campus, 18(1), 16-20.

Al-Mukhaini, E., Al-Qayoudhi, W., \& Al-Badi, A. (2014). Adoption of Social Networking in Education: A Study of the Use of Social Networks by Higher Education Students in Oman. Journal of International Education Research, 10(2), 143-154.

Amirault, R. (2012). Distance Learning in the 21st Century University: Key Issues for Leaders and Faculty. Quarterly Review of Distance Education, 13(4), 253265.

Aragón, A., AlDoubi, S., Kaminski, K., \& Anderson, S. (2014). Social Networking: Boundaries and Limits. TechTrends: Linking Research and Practice to Improve Learning, 58(2), 25-31.

ICONO14 | Año 2015 Volumen 13 Nº 2 | ISSN: 1697-8293 | DOI: ri14.v13i2.788 
Araujo, U., Fruchter, R., Garbín, M.C, Nóbilo, L., \& Arantes, V. (2014). The reorganization of time, space, and relationships in school with the use of active learning methodologies and collaborative tools. ETD: Educaçao Temática Digital, 16(1), 84-99.

Balakrishnan, V. (2014). Using Social Networks to Enhance Teaching and Learning Experiences in Higher Learning Institutions. Innovations in Education and Teaching International, 51(6), 595-606.

Barrientos, J., \& Mogollón, E. (2013). Estrategias interactivas de aprendizajes matemáticos con el uso de las tecnologías de información y comunicación para estudiantes de educación media general. REDHECS: Revista electrónica de Humanidades, Educación y Comunicación Social, 8(14), 24-39.

Barker, V. (2012). A Generational Comparison of Social Networking Site Use: The Influence of Age and Social Identity. International Journal of Aging and Human Development, 74(2), 163-187.

Cabero-Almenara, J., \& Marín, V. (2014). Posibilidades educativas de las redes sociales y el trabajo en grupo. Percepciones de los alumnos universitarios. Comunicar: Revista científica iberoamericana de comunicación y educación, 42, 165-172.

Cappella, E., Neal, J., \& Sahu, N. (2012). Children's Agreement on Classroom Social Networks: Cross-Level Predictors in Urban Elementary Schools. Journal of Developmental Psychology, 58(3), 285-313.

Castejón, F.J., Santos, M., \& Palacios, A. (2013) Cuestionarios obremetodología y evaluación en formación inicial en EducaciónFísica.Revista Internacional de Medicina y Ciencias de la Actividad Física y el Deporte, 12(1), 1-23.

Cohn, P. J. (1991). An exploratory study on peak performance in golf. The Sport Psychologist, 5, 1-14.

Corbetta, P. (2007). Metodologías y técnicas de investigación social. Madrid: McGrawHill

Denzin, N. K., \& Lincoln, Y. S. (1994). Handbook of Qualitative Research. Thousand Oaks, CA: Sage.

English, T., \& Carstensen, L. (2014). Selective Narrowing of Social Networks across Adulthood is Associated with Improved Emotional Experience in Daily Life. International Journal of Behavioral Development, 38(2), 195-202.

Fernández-Delgado, F.C. (2012). La riqueza de las redes en la educación universitaria. 
Teoría de la Educación: Educación y Cultura en la Sociedad de la Información, 13(2), 200-219.

García-Valcárcel, A., Muñoz-Repiso, A., Hernández-Martín, A. \& Recamán, A. (2012). La metodología del aprendizaje colaborativo a través de las TIC: una aproximación a las opiniones de profesores y alumnos. Revista complutense de educación, 23(1), 161-188.

García, A., \& Martín, A. (2013). Aplicando el EEES en la Universidad española: un estudio de caso sobre la utilización de metodologías 2.0 en las nuevas titulaciones de grado. Historia y comunicación social, 18(1), 603-613.

García-González, J. (2014). Evaluación educativa de los estudiantes en una red social en línea. Un estudio en la educación superior. Revista Iberoamericana de educación, 64,127-141.

Jacquemin, S., Smelser, L., \& Bernot, M. (2014). Twitter in the Higher Education Classroom: A Student and Faculty Assessment of Use and Perception. Journal of College Science Teaching, 43(6), 22-27.

Libarkin, J. C., \& Kurdziel. J. P. (202). Research methodologies in science education: Qualitative data. Journal of Geoscience Education, 50, 195-200.

Lincoln, Y. S., \& Guba, E. (1985). Naturalistic inquiry. Newbury Park, CA: Sage.

Low, S., Polanin, J., \& Espelage, D. (2013). The Role of Social Networks in Physical and Relational Aggression among Young Adolescents. Journal of Youth and Adolescence, 42(7), 1078-1089.

Lu, J., \& Churchill, D. (2014). The Effect of Social Interaction on Learning Engagement in a Social Networking Environment. Interactive Learning Environments, 22(4), 401-417.

Madill, R., Gest, S., \& Rodkin, P. (2014). Measuring Teacher Knowledge of Classroom Social Networks: Convergent and Predictive Validity in Elementary School Classrooms. Society for Research on Educational Effectiveness,11(2), 12-21.

Marín, V., Salinas, J., \& De Benito, B. (2014). Research Results of Two Personal Learning Environments Experiments in a Higher Education Institution. Interactive Learning Environments, 22(2), 205-220.

Martínez- González, F. (2013). Los trabajos tutorizados como método de aprendizaje a través de la investigación y la aplicación de las nuevas tecnologías. Ariadna: cultura, educación y tecnología, 1(1), 53-57.

Martínez-Berruezo, M.A., \& García-Varela, A.B. (2013). Análisis de la influencia de 
la virtualización en la motivación del alumnado universitario de primer curso de Magisterio. Revista de educación, 362, 42-68.

Monsalve, J.C, \& Granada, L.A. (2013). Redes sociales: aproximación a un estado del arte. Lámpsakos, 9, 34-41.

Moral, M.E., \& Villalustre, L. (2012). Presencia de los futuros maestros en las redes sociales y perspectivas de uso educativo. RELATEC: Revista Latinoamericana de Tecnología Educativa, 11(1), 41-51.

Ormart, E.R. \& Navés, F.V. (2014). El uso de redes sociales como soporte educativo. Revista CPU-e, 18, 162-171.

Patton, M. Q. (2002). Qualitative Research and Evaluation Methods. Thousand Oaks, CA: Sage.

Pérez-Fernández, F., \& Vílchez, J.E. (2013). Percepción de futuros maestros sobre el potencial de las TIC en la educación de las expectativas a la realidad. Fuentes: Revista de la Facultad de Ciencias de la Educación, 13, 155-152.

Román, M., Cardemil, C., \& Carrasco, A. (2012). Enfoque y metodología para evaluar la calidad del proceso pedagógico que incorpora TIC en el aula. Revista Iberoamericana de Evaluación Educativa, 4(2), 8-35.

Saldaña, J. (2009). The Coding Manual for Qualitative Researchers. Thousand Oaks, CA: Sage.

Schram, A. (2014). A Mixed Methods Content Analysis of the Research Literature in Science Education. International Journal of Science Education, 36(15), 26192638.

Smith, J. A. \& Osborne, M. (2003). Interpretative Phenomenological Analysis. In J.A. Smith (Ed.) Qualitative psychology: A practical guide to research methods. (2nd ed., pp. 53-79). London: Sage

Toetenel, L. (2014). Social Networking: A Collaborative Open Educational Resource. Computer Assisted Language Learning, 27(2), 149-162.

Waldrip, B. et al. (2014). Student Perceptions of Personalised Learning: Development and Validation of a Questionnaire with Regional Secondary Students. Learning Environments Research, 17(3), 355-370.

Windes, D., \& Lesht, F. (2014). The Effects of Online Teaching Experience and Institution Type on Faculty Perceptions of Teaching Online. Online Journal of Distance Learning Administration, 17(1), 1-12. 